\section{Genome Resources of Three West African Strains of Pantoea ananatis Causing Bacterial Blight and Grain Discoloration of Rice}

\author{
Kossi Kini, ${ }^{1,2}$ Pierre Lefeuvre, ${ }^{3}$ Lucie Poulin, ${ }^{2}$ Drissa Silué, ${ }^{1}$ and Ralf Koebnik ${ }^{2, \dagger}$ \\ ${ }^{1}$ AfricaRice, Plant Pathology, Africa Rice Center, B.P. 2031, Cotonou, Benin \\ ${ }^{2}$ IRD, Cirad, Université de Montpellier, IPME, 34394 Montpellier, France \\ ${ }^{3}$ CIRAD, UMR PVBMT, F-97410 St. Pierre, La Réunion, France
}

\begin{abstract}
Members of the genus Pantoea have been reported as pathogens for many economically important crops, including rice. Little is known about their host-pathogen interactions at the molecular level and the lack of comprehensive genome data impedes targeted breeding strategies toward resistant rice cultivars. Here, we describe the structural and functional annotation of the draft genome sequences of three rice-pathogenic Pantoea ananatis strains, ARC272, ARC310, and ARC311, which were isolated in Burkina Faso, Togo, and Benin, respectively. The genome sequences of these strains will help in developing molecular diagnostic tools and provide new insight into common traits that may enable $P$. ananatis to infect rice.
\end{abstract}

\section{Resource Announcement}

Bacteria of the species Pantoea ananatis are implicated in diseases of a wide range of economically important crops, such as rice, maize, sorghum, and onion (Achbani et al. 2015; Cortesi and Pizzatti 2007; Cota et al. 2010; Egorova et al. 2015; Gitaitis and Gay 1997; Mondal et al. 2011; Yan et al. 2010) and are a serious threat for crop production across the globe (Coutinho and Venter 2009; Walterson and Stavrinides 2015; Weller-Stuart et al. 2017). $P$. ananatis was recently reported as being responsible for bacterial leaf blight of rice in several countries of sub-Saharan Africa, such as Benin, Burkina Faso, Mali, Nigeria, Senegal, and Togo (Kini et al. 2017a, b, 2018). Comparative genome sequence analyses revealed candidate pathogenicity determinants of Asian and American strains of $P$. ananatis (Asselin et al. 2018; De Maayer et al. 2011, 2014; Stice et al. 2018). To complement these data, we report the first genome sequences of West African $P$. ananatis strains, which were isolated from rice leaves or seeds in Benin, Burkina Faso, and Togo.

Bacteria were isolated during surveys carried out from 2010 to 2013 in several rice fields in West Africa. Two strains were isolated from leaves with symptoms resembling bacterial blight and one strain was isolated from a discolored rice grain (Table 1). Pure cultures were obtained using the protocol described by Kini et al. (2017a). Genomic DNA was extracted from the cultures using the Wizard genomic DNA purification kit of Promega (Madison, WI, U.S.A.) according to the manufacturer instructions. The genomes were sequenced using the Illumina

Current address of L. Poulin: Laboratoire de Biologie et Pathologie Végétales, EA 1157, SFR 4207 QUASAV, UFR Sciences et Techniques, Université de Nantes, 44322 Nantes, France.

${ }^{\dagger}$ Corresponding author: R. Koebnik; koebnik@gmx.de

*The $e$-Xtra logo stands for "electronic extra" and indicates that one supplementary table is published online.

The author(s) declare no conflict of interest.

Accepted for publication 23 April 2020.
e-Xtra*

Funding

This study was supported by a Ph.D. scholarship for K. Kini from the French Research Institute for Sustainable Development (IRD) within the programme "Allocation de Recherche pour une Thèse au Sud" (ARTS). The Africa Rice Center (AfricaRice) and IRD received financial support from the Global Rice Science Partnership (GRiSP). In addition, the Ministry of Foreign Affairs, Japan, contributed to the project.

\section{Keywords}

bacteriology, Erwiniaceae, genome sequence, Pantoea ananatis, rice pathogen, West Africa 
Table 1. Project information and genomic features of Pantoea ananatis strains ARC272, ARC310, and ARC311

\begin{tabular}{lccc} 
Strain & ARC272 & ARC310 & ARC311 \\
Sample & Rice leaf & Rice leaf & Rice seed \\
Origin of sample & Burkina Faso: Bobo-Dioulasso, Bama & Togo: Deve & Benin \\
Collection date & 22 Oct 2013 & 29 Jun 2010 & 2013 \\
GenBank BioProject & PRJNA399058 & PRJNA399062 & PRJNA399063 \\
GenBank BioSample & SAMN07522540 & SAMN07522547 & SAMN07522548 \\
GenBank WGS project & PUGD00000000 & PUGC00000000 & PUGB00000000 \\
Coverage & $62 \times$ & $90 \times$ & $73 \times$ \\
Contigs & 128 & 216 & 166 \\
N50 & 72,412 & 36,729 & 47,567 \\
L50 & 21 & 40 & 32 \\
G+C content (\%) & 53.50 & 53.59 & 53.58 \\
Genome size (bp) & $4,615,013$ & $4,598,241$ & $4,593,415$ \\
Predicted genes & 4,471 & 4,470 & 4,420 \\
Protein-coding genes & 4,258 & 4,234 & 4,180 \\
RNA genes & 104 & 105 & 107 \\
Pseudo genes & 109 & 131 & 133 \\
\hline
\end{tabular}

HiSeq 2500 platform (Fasteris SA, Geneva, Switzerland). Nextera-XT libraries (Illumina) with an insert size of $250 \mathrm{bp}$ to $1.5 \mathrm{~kb}$ were generated and 125 sequencing cycles were performed from both sides. The shotgun sequencing yielded paired-end reads for $1,827,519$ clusters (457 Mb) for strain ARC272, 2,690,980 clusters (673 Mb) for strain ARC310, and 2,211,169 clusters (553 $\mathrm{Mb})$ for strain ARC311. Upon adapter removal, reads were assembled using the Edena algorithm v3.131028 (Hernandez et al. 2013). Contigs were annotated with GeneMarkS+ (revision 4.4) (Borodovsky and Lomsadze 2014), as implemented in the NCBI Prokaryotic Genome Annotation Pipeline (https://www.ncbi.nlm.nih.gov/genome/annotation_prok/). The three draft genome sequences were each 4.6 Mbp in size and had an average $\mathrm{G}+\mathrm{C}$ content of about $53.5 \%$ (Table 1). Between 4,420 and 4,471 genes were predicted for the three genomes, among which $95 \%$ were predicted to encode proteins (Table 1 ).

Genome-wide average nucleotide identities were calculated at http://enve-omics.ce. gatech.edu (Rodriguez-R and Konstantinidis 2016) and confirmed the taxonomic position of the three strains (Goris et al. 2007). Genome sequences of all three strains were $>99 \%$ identical to those from $P$. ananatis strains that were collected from rice in China (strains PaMB1, RSA47, and Sd-1), India (strains NS296, NS303, and NS311), or Spain (strains 1.38 and AMG521) (Megías et al. 2016, 2018; Midha et al. 2016), and clearly distinct from those of the two other major plant-pathogenic Pantoea species, $P$. agglomerans and $P$. stewartii (Supplementary Table S1). Interestingly, the species $P$. ananatis forms two major clades, one of which apparently consists only of isolates originating from rice-associated samples, such as leaves, seeds, or the rhizosphere whereas the other clade includes strains from diverse origin, such as plants (e.g., eucalyptus, grasses, maize, onion, pineapple, strawberry, and sugarcane), but also insects and environmental samples. The three West African strains from this study belong to the rice-associated clade of $P$. ananatis. With this expanded geographic coverage, our new data sets represent a valuable resource for the functional analysis of genomic traits that might be involved in host range adaptation of $P$. ananatis.

Draft genome sequences of the three strains ARC272, ARC310, and ARC311 were deposited at NCBI GenBank under BioProject accession numbers PRJNA399058, PRJNA399062, and PRJNA399063, respectively. The three bacterial strains described in this study are available from the Africa Rice Center (http://www.africarice.org).

\section{Literature Cited}

Achbani, E. H., Mazouz, H., Benbouazza, A., and Sadik, S. 2015. First detection of Pantoea ananatis, the causal agent of bacterial center rot of onion in Morocco. J. Crop Prot. 5:11-17.

Asselin, J. A. E., Bonasera, J. M., and Beer, S. V. 2018. Center rot of onion (Allium cepa) caused by Pantoea ananatis requires pepM, a predicted phosphonate-related gene. Mol. Plant-Microbe Interact. 31:1291-1300.
Borodovsky, M., and Lomsadze, A. 2014. Gene identification in prokaryotic genomes, phages, metagenomes, and EST sequences with GeneMarkS suite. Curr. Protoc. Microbiol. 32:1E.7.1-1E.7.17.

Cortesi, P., and Pizzatti, C. 2007. Palea browning, a new disease of rice in Italy caused by Pantoea ananatis. J. Plant Pathol. 89:76.

Cota, L. V., Costa, R. V., Silva, D. D., Parreira, D. F., Lana, U. G. P., and Casela, C. R. 2010. First report of pathogenicity of Pantoea ananatis in sorghum (Sorghum bicolor) in Brazil. Australas. Plant Dis. Notes 5:120-122. 
Coutinho, T. A., and Venter, S. N. 2009. Pantoea ananatis: An unconventional plant pathogen. Mol. Plant Pathol. 10:325-335.

De Maayer, P., Chan, W. Y., Rubagotti, E., Venter, S. N., Toth, I. K., Birch, P. R. J., and Coutinho, T. A. 2014. Analysis of the Pantoea ananatis pan-genome reveals factors underlying its ability to colonize and interact with plant, insect and vertebrate hosts. BMC Genomics 15:404.

De Maayer, P., Venter, S. N., Kamber, T., Duffy, B., Coutinho, T. A., and Smits, T. H. 2011. Comparative genomics of the type VI secretion systems of Pantoea and Erwinia species reveals the presence of putative effector islands that may be translocated by the VgrG and Hcp proteins. BMC Genomics 12:576.

Egorova, M., Mazurin, E., and Ignatov, A. N. 2015. First report of Pantoea ananatis causing grain discolouration and leaf blight of rice in Russia. New Dis. Rep. 32:21.

Gitaitis, R. D., and Gay, J. D. 1997. First report of a leaf blight, seed stalk rot, and bulb decay of onion by Pantoea ananas in Georgia. Plant Dis. 81:1096.

Goris, J., Konstantinidis, K. T., Klappenbach, J. A., Coenye, T., Vandamme, P., and Tiedje, J. M. 2007. DNA-DNA hybridization values and their relationship to wholegenome sequence similarities. Int. J. Syst. Evol. Microbiol. 57:81-91.

Hernandez, D., Tewhey, R., Veyrieras, J. B., Farinelli, L., Østerås, M., François, P., and Schrenzel, J. 2013. De novo finished 2.8 Mbp Staphylococcus aureus genome assembly from $100 \mathrm{bp}$ short and long range paired-end reads. Bioinformatics 30 : 40-49.

Kini, K., Agnimonhan, R., Afolabi, O., Soglonou, B., Silué, D., and Koebnik, R. 2017a. First report of a new bacterial leaf blight of rice caused by Pantoea ananatis and Pantoea stewartii in Togo. Plant Dis. 101:241.

Kini, K., Agnimonhan, R., Afolabi, T., Milan, B., Soglonou, B., Gbogbo, V., Koebnik, R., and Silué, D. 2017b. First report of a new bacterial leaf blight of rice caused by Pantoea ananatis and Pantoea stewartii in Benin. Plant Dis. 101:242.
Kini, K., Agnimonhan, R., Dossa, R., Silué, D., and Koebnik, R. 2018. A diagnostic multiplex PCR scheme for identification of plant-associated bacteria of the genus Pantoea. bioRxiv 456806.

Megías, E., Dos Reis, F. B., Jr., Ribeiro, R. A., Ollero, F. J., Megías, M., and Hungria, M. 2018. Draft genome sequence of Pantoea ananatis strain 1.38 , a bacterium isolated from the rhizosphere of Oryza sativa var. Puntal that shows biotechnological potential as an inoculant. Genome Announc. 6:e01547-e17.

Megías, E., Megías, M., Ollero, F. J., and Hungria, M. 2016. Draft genome sequence of Pantoea ananatis strain AMG521, a rice plant growth-promoting bacterial endophyte isolated from the Guadalquivir Marshes in Southern Spain. Genome Announc. 4:e01681-e15.

Midha, S., Bansal, K., Sharma, S., Kumar, N., Patil, P. P., Chaudhry, V., and Patil, P. B. 2016. Genomic resource of rice seed associated bacteria. Front. Microbiol. 6:1551.

Mondal, K. K., Mani, C., Singh, J., Kim, J. G., and Mudgett, M. B. 2011. A new leaf blight of rice caused by Pantoea ananatis in India. Plant Dis. 95:1582.

Rodriguez-R, L. M., and Konstantinidis, K. T. 2016. The enveomics collection: A toolbox for specialized analyses of microbial genomes and metagenomes. PeerJ Preprints 4:e1900v1.

Stice, S. P., Stumpf, S. D., Gitaitis, R. D., Kvitko, B. H., and Dutta, B. 2018. Pantoea ananatis genetic diversity analysis reveals limited genomic diversity as well as accessory genes correlated with onion pathogenicity. Front. Microbiol. 9:184.

Walterson, A. M., and Stavrinides, J. 2015. Pantoea: Insights into a highly versatile and diverse genus within the Enterobacteriaceae. FEMS Microbiol. Rev. 39:968-984.

Weller-Stuart, T., De Maayer, P., and Coutinho, T. 2017. Pantoea ananatis: Genomic insights into a versatile pathogen. Mol. Plant Pathol. 18:1191-1198.

Yan, H., Yu, S. H., Xie, G. L., Fang, W., Su, T., and Li, B. 2010. Grain discoloration of rice caused by Pantoea ananatis (synonym Enwinia uredovora) in China. Plant Dis. 94:482. 